\title{
ASPECTOS HIDROGEOLÓGICOS EN EL SITIO DEL EMBALSE CIPRESES, P.H. CARIBLANCO Y APLICACIÓN DE TRAZADORES EN AGUA SUBTERRÁNEA
}

\author{
Asdrúbal Vargas ${ }^{1} \&$ José Francisco Fernández ${ }^{2}$ \\ ${ }^{1}$ C. S. Exploración Subterránea ${ }^{2}$ C.S. Estudios Básicos de Ingeniería \\ Instituto Costarricense de Electricidad \\ E-Mail: avargass@ice.go.cr / jffernandez@ice.go.cr
}

\begin{abstract}
The hydrogeological conditions in an area between Cariblanco and San Miguel of Sarapiquí on the northeastern slope of Cerro Congo was investigated. The area is characterized by volcanic rocks and high precipitation values. Some lava flows have moderate permeability and generate important springs; for example, the spring San Miguel with a discharge greater than 300 1/s. Near to the spring the Instituto Costarricense de Electricidad (ICE) has planned to built a reservoir and a dike for the Cariblanco hydroelectric project. The objective of this work is to evaluate the incidence of the excavation over the spring San Miguel.

Two water table aquifers and a confined aquifer exist in the study area. The upper water table aquifer is formed by fractured lavas and the groundwater flows to the surface mainly through the San Miguel spring. By using hydrochemical analysis, geological core information and artificial tracer injected in groundwater the absence of the water table aquifer in the reservoir area was defined and the construction of the water reservoir probably has no influence on the discharge and water quality of the San Miguel spring. Artificial tracers can be used to simulate the flow and transport of contaminant and to calculate the mechanical dispersion coefficient of some solutes in the groundwater.
\end{abstract}

RESUMEN: Se investigaron las condiciones hidrogeológicas en una zona ubicada en la falda noreste del cerro Congo, entre el poblado de Cariblanco y San Miguel de Sarapiquí. Se evaluó la influencia de la construcción del embalse Cipreses del P.H. Cariblanco que pretende construir el Instituto Costarricense de Electricidad, sobre el manantial San Miguel que abastece el acueducto del poblado homónimo aplicando trazadores artificiales. En el área de estudio existen al menos tres acuíferos, dos de ellos acuíferos libres y uno confinado en lavas fracturadas. El acuífero libre superior está formado por lavas fracturadas y da origen al manantial San Miguel. Este manantial es producto de la descarga de agua subterránea debido a la culminación del acuífero y a que está limitado en la base por material menos permeable lo cual induce al agua a salir a la superficie.

Por medio de varios ensayos de trazador y conociendo la disposición estratigráfica y espacial del acuífero libre en lavas, se puede afirmar que la excavación del embalse Cipreses no tendrá influencia sobre el manantial San Miguel.

\section{INTRODUCCIÓN}

La zona de estudio se ubica en la hoja topográfica Poás, en las coordenadas 252000252800 y $514000-5148000$ (Fig. 1). Cercanas a la zona de estudio existen varias lagunas, entre ellas Laguna Hule, Congo y María Aguilar. Esta zona se encuentra en las faldas del cerro Congo a una altura de 900 m s.n.m, casi 1000 m por debajo de la cima. 


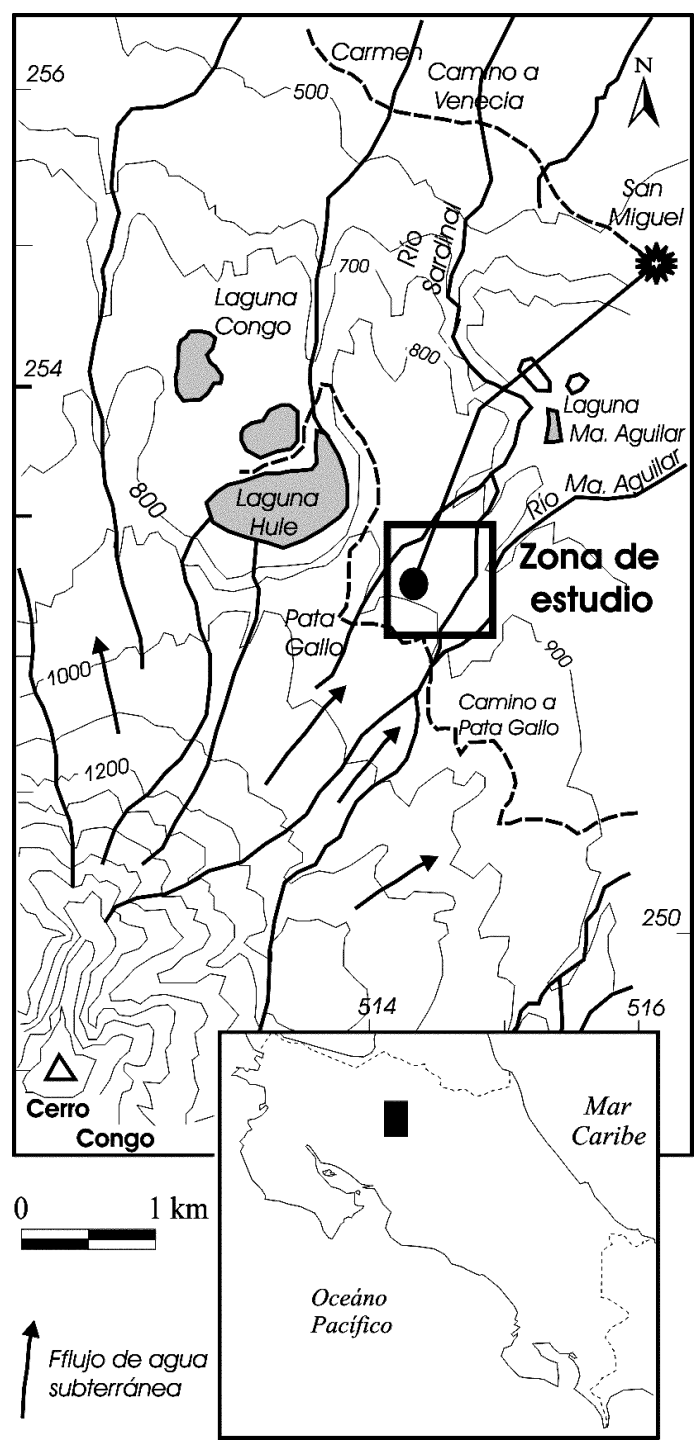

Fig. 1: Ubicación de la zona de estudio en parte de la hoja topográfica, Poás.

En la zona de estudio existe un manantial que abastece el acueducto de San Miguel de Sarapiquí. Cerca de este punto se pretende construir el embalse del P.H. Cariblanco para almacenar agua; sin embargo, es necesario evaluar cual podría ser la influencia de la excavación sobre el manantial. En éste trabajo se pretende investigar las condiciones hidrogeológicas del sitio para construir un modelo hidrogeológico conceptual y evaluar la influencia de la construcción del embalse sobre el manantial San Miguel.
Se contó con información de dos perforaciones con recuperación de testigos, análisis hidroquímicos, ensayos de trazadores y pruebas tipo "slug test". Además, se cuenta con información hidrológica del Centro de Estudios Básicos del Instituto Costarricense de Electricidad.

La investigación de las condiciones hidrogeológicas se puede llevar a cabo por medio del análisis de la hidrogeoquímica (Custodio \& Llamas, 1983) o por medio del empleo de trazadores artificiales (Plata, 1995). Se pretende mostrar que los trazadores artificiales son una herramienta hidrogeológica que permite determinar la orientación del flujo de agua subterránea en acuíferos relativamente someros. Además, se pueden utilizar para establecer el tipo de relación que existe entre varios acuíferos. Entre los trazadores artificiales más utilizados se encuentra el cloruro de sodio $(\mathrm{NaCl})$, que, utilizado en cantidades adecuadas y con un procedimiento detallado, es inocuo ambientalmente y no causa problemas a la salud humana. La implementación de este trazador se puede realizar en perforaciones de pequeño diámetro por medio de una manguera y se puede detectar con conductivímetros de campo por lo que su aplicación brinda múltiples oportunidades en la auscultación hidrogeológica de sitios elegidos para la construcción de obras civiles.

\section{ASPECTOS HIDROLÓGICOS}

La alta pluviosidad de la zona está registrada en la estación pluviográfica quebrada Gata del ICE, ubicada a $7 \mathrm{~km}$ al suroeste de la zona de estudio. En el año 1999 el total de precipitación fue de $6312 \mathrm{~mm}$ mientras que para el año 2000 fue de $5249 \mathrm{~mm}$, superando el valor promedio de precipitación anual a nivel nacional en más de 3000 mm (Fig. 2), considerándose la zona como de alta pluviosidad. Los meses de mayor precipitación registrados son enero, noviembre y diciembre, seguidos por mayo, junio y julio, mientras que marzo y abril son los meses más secos.

Entre los cuerpos de agua superficial más importantes en la zona de estudio destacan los ríos María Aguilar y Sardinal, y las quebradas Sanguijuela y Naciente, que tienen una dirección 


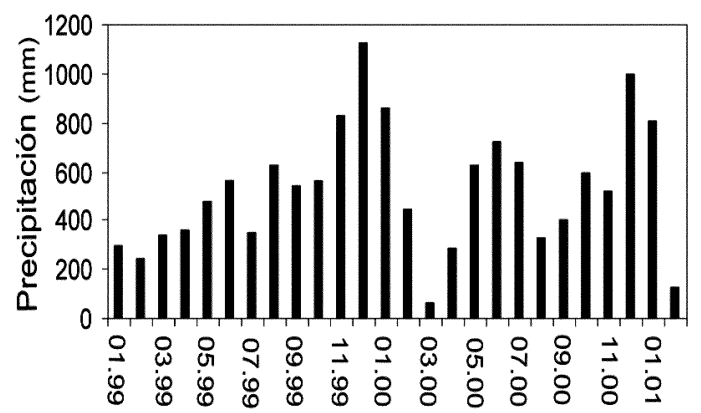

Fig. 2: Histograma de precipitación media mensual en la Estación de la quebrada Gata.

Fuente: Centro de Servicio Estudios Básicos de Ingeniería del ICE.

noreste y forman un patrón de drenaje dendrítico a subdendrítico, típico de zonas ubicadas alrededor de conos volcánicos relativamente jóvenes.

\section{Quebrada Naciente}

Es producto del manantial San Miguel, que es captado en parte para abastecer el acueducto de San Miguel de Sarapiquí y para actividades ganaderas de algunas fincas de la zona, fluyendo por la quebrada un caudal de alrededor de 250 1/s (Cuadro 1). Hay un incremento del caudal entre el sitio 1 y el 3 debido a la descarga de agua de una tubería que capta agua directamente del manantial.

Cuadro 1

Caudales de la quebrada Naciente aforados el 28 de agosto y el 12 de setiembre del 2001

\begin{tabular}{cccc}
\hline Aforo & Sitio & Lugar & Caudal (1/s) \\
\hline 28 /08/2001 & & & \\
1 A & Sitio 1 & 200 m de toma & 248,3 \\
1 B & Sitio 1 & & 245,1 \\
3 A & Sitio 3 & $600 \mathrm{~m}$ de toma & 280,0 \\
3 B & Sitio 3 & & 292,0 \\
\hline 12/09/2001 & & & \\
1 A & Sitio 1 & 200 m de toma & 255,4 \\
2 A & Sitio 2 & $400 \mathrm{~m}$ de toma & 276,8 \\
3 A & Sitio 3 & $600 \mathrm{~m}$ de toma & 295,0 \\
\hline
\end{tabular}

Fuente: Estudios Básicos de Ingeniería, ICE.

\section{Quebrada Sanguijuela}

Tiene su origen en un punto relativamente cercano a la quebrada Naciente con un caudal promedio de 70 1/s (Cuadro 2). No obstante su cercanía, los análisis químicos realizados no muestran una clara relación entre el manantial San Miguel y la quebrada Sanguijuela.

Cuadro 2

Aforos de la quebrada Sanguijuela realizados en setiembre de 2001

\begin{tabular}{cccc}
\hline Aforo & Sitio & Caudal (1/s) & $\begin{array}{c}\text { Caudal promedio } \\
(1 / \mathrm{s})\end{array}$ \\
\hline 1 A & Sitio 1 & 71,9 & \\
1 B & Sitio 1 & 70,9 & 71,4 \\
2 A & Sitio 2 & 70,5 & \\
2 B & Sitio 2 & 65,1 & 67,8 \\
3 A & Sitio 3 & 80,1 & \\
3 B & Sitio 3 & 72,6 & 76,4 \\
\hline
\end{tabular}

\section{ASPECTOS HIDROGEOLÓGICOS}

Las condiciones de alta pluviosidad, existencia de materiales permeables así como pendientes pronunciadas favorecen el almacenamiento de volúmenes importantes de agua subterránea en reservorios denominados acuíferos. En el área de estudio se han identificado al menos tres acuíferos, dos de ellos constituido por lavas. Estos acuíferos son recargados por la precipitación y la dirección del agua subterránea es noreste.

\section{Acuífero libre en lavas}

Esta formado por lavas fracturadas con porosidad primaria por vesículas y secundaria por fracturamiento, estando cubierto en la parte superior por una arcilla roja de baja permeabilidad que forma parte de un coluvio. Este acuífero aparece en la perforación 37 PHC a una profundidad de $20,7 \mathrm{~m}$ y tiene un espesor de $29 \mathrm{~m}$. Debajo del acuífero aparecen arenas de menor permeabilidad. En el registro litológico de dicha perforación es posible observar planos de fracturas cubiertos de óxidos de hierro y manganeso de 
color gris, lo que indica la presencia de un flujo de agua. En la perforación 40 PHC no se ha encontrado evidencias de este acuífero.

Este acuífero es de tipo libre con un nivel de agua subterránea que se encuentra a una profundidad de $27,3 \mathrm{~m}$ y la dirección de flujo tiene una orientación de suroeste hacia el noreste. El gradiente hidráulico en la zona terminal del acuífero es de 0,03 , obtenido a partir de la elevación del nivel freático en la perforación 37 PHC que es de 867,7 m s.n.m., la elevación del manantial San Miguel estimada en 865,0 m s.n.m y la distancia entre la perforación y el manantial calculada en $78,8 \mathrm{~m}$. La permeabilidad aparente del acuífero es de moderada a alta y se estima una extensión lateral promedio de $200 \mathrm{~m}$.

\section{Acuífero libre en arenas}

Se nota su presencia en la perforación 40 PHC, desde la superficie hasta $16 \mathrm{~m}$ de profundidad, constituido por arenas finas y medias de color negro o café. Su permeabilidad aparente es media, con una conductividad hidráulica de 1 $\times 10^{-3} \mathrm{~cm} / \mathrm{s}$ medida por medio de un ensayo "slug test". Además, por medio de un análisis granulométrico se logró estimar una permeabilidad aproximada de $7 \times 10^{-2} \mathrm{~cm} / \mathrm{s}$. El nivel del agua se encuentra a $1 \mathrm{~m}$ de profundidad constituyéndose en un acuífero libre, el cual posiblemente se encontraría durante la excavación del embalse.

\section{Acuífero confinado en lavas}

Está limitado en el techo por una arcilla café de baja permeabilidad de varios metros de espesor y está constituido por un material lávico con porosidad primaria por vesículas y fracturamiento. El agua subterránea dentro del acuífero presenta confinamiento a tal grado que en la perforación 40 PHC el agua es surgente en la superficie. Tiene un espesor de alrededor de $13 \mathrm{~m}$ y una permeabilidad aparente de moderada a alta. La extensión lateral no está aún bien definida.

\section{Manantiales}

En el área de estudio se han identificado al menos tres descargas de agua subterránea con caudales que varían de $0,5 \mathrm{l} / \mathrm{s}$ hasta $400 \mathrm{l} / \mathrm{s}$. El manantial de mayor caudal es el denominado San Miguel el cual abastece el poblado de San Miguel de Sarapiquí y se encuentra a una elevación de 865 m s.n.m. Este manantial se encuentra a $300 \mathrm{~m}$ del borde de la excavación del embalse denominado Cipreses (Fig. 3). El caudal de este manantial no se ha aforado en su totalidad, sin embargo, se cuenta con datos de caudales del remanente de la toma del acueducto San Miguel (Cuadro 1), lo que indica un caudal superior a 300 1/s. Parte del agua del manantial es captado por una estructura de concreto con escasa protección contra la contaminación.

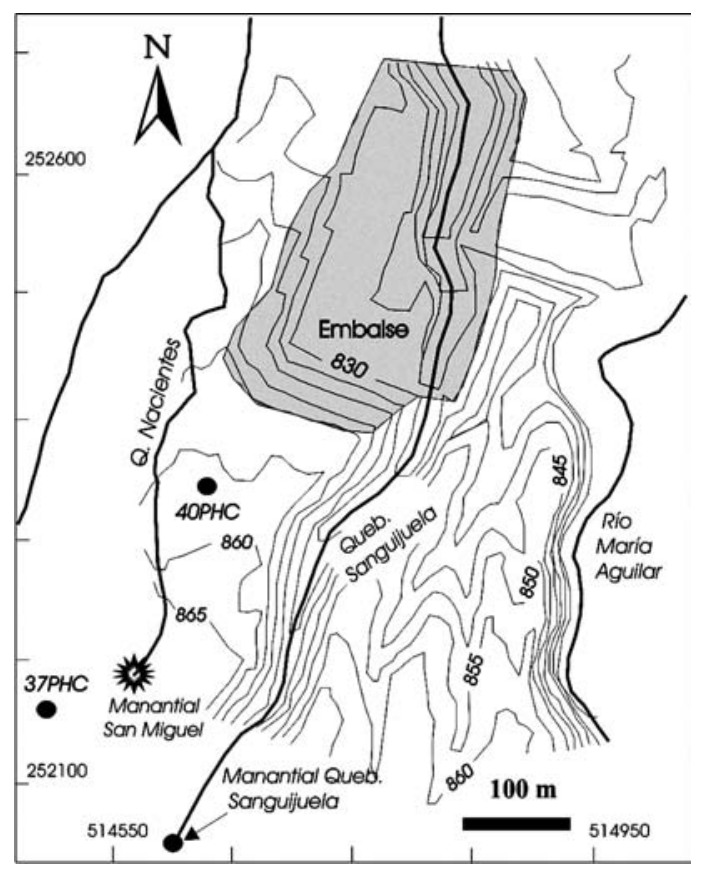

Fig. 3: Ubicación del embalse Cipreses, manantial San Miguel y las perforaciones $37 \mathrm{PHC}$ y $40 \mathrm{PHC}$.

\section{HIDROGEOQUÍMICA}

Para dar una respuesta a las interrogantes planteadas con motivo de la probable excavación del embalse Cipreses y la relación con los 
acuíferos de la zona se realizaron análisis químicos de muestras de agua provenientes de manantiales y de quebradas. Además, se ejecutaron tres ensayos de inyección de un trazador en el agua subterránea por medio de una perforación de pequeño diámetro.

Para caracterizar y establecer posibles relaciones entre acuíferos, quebradas y manantiales se realizaron análisis físico químicos de agua de varios puntos de la zona entre los cuales se encuentra el manantial San Miguel, las quebradas Sanguijuela, Sardinal, Naciente 1 y 2, la perforación 40 y el río María Aguilar (Cuadro 3). Se determinaron, entre otras cosas, las concentraciones de iones mayoritarios, para luego construir los respectivos diagramas de Stiff y establecer posibles facies hidrogeoquímicas en la zona de estudio.

El día 12 de setiembre del 2001 se recolectaron muestras de agua de cuatro puntos correspondientes a la quebrada Sanguijuela, río María Aguilar, naciente San Miguel y quebrada Sardinal y se analizaron las concentraciones de iones ma- yores incluyendo sílice y conductividad (Cuadro 4), por medio de los procedimientos establecidos por Anónimo (1985). En la quebrada Sanguijuela se nota un valor de nitratos superior a los otros puntos de muestreo. En los diagramas de Stiff (Fig. 4) se observan ligeras diferencias entre las concentraciones de iones en el agua de la quebrada Sanguijuela y del río María Aguilar y aún más leves con el manantial San Miguel. En la quebrada Sardinal la concentración de bicarbonato es mayor que en las otras muestras.

Entre martes 9 y miércoles 10 de octubre se recolectaron nuevamente muestras de agua de cinco puntos correspondientes a la naciente San Miguel (M1), quebrada Tajo Naciente No. 1 (M2), quebrada No. 2 (M3), quebrada Sanguijuela (M4)y la quebrada Tajo Naciente No. 2 (M5) (Cuadro 5). Los resultados de los análisis muestran una relación entre el agua de los nacientes con el manantial San Miguel, pero no es muy evidente con la quebrada Sanguijuela. Esta última muestra una concentración de $33 \mathrm{mg} / \mathrm{l}$ de bicarbonato;

Cuadro 3

Puntos de muestreo de agua para análisis físico-químico

\begin{tabular}{ccccc}
\hline Punto Muestreo & Latitud & Longitud & Elevación (ms.n.m) & Caudal (1/s) \\
\hline Naciente San Miguel & 252187 & 514369 & 865 & 300 \\
Perf. 40 & 252345 & 514628 & 856 & - \\
Quebrada 2 & 252267 & 514457 & 865 & 1 \\
Q. Sanguijuela & 252035 & 514602 & 920 & 0,5 \\
Q. Tajo Naciente 1 & 252100 & 513900 & 921 & 3 \\
Q. Tajo Naciente 2 & 252100 & 513900 & 921 & 5 \\
Río María Aguilar & 252200 & 513800 & 820 & - \\
\hline
\end{tabular}

Cuadro 4

Características químicas del agua en cuatro puntos de muestreo

\begin{tabular}{lcccc}
\hline Parámetro & M1 & M2 & M3 & M4 \\
\hline Sodio (mg/l) & 4,33 & 3,95 & 4,92 & 4,86 \\
Potasio (mg/l) & 3,40 & 2,37 & 2,69 & 2,24 \\
Calcio (mg/l) & 10,6 & 9,08 & 10,7 & 11,3 \\
Magnesio (mg/l) & 2,31 & 2,44 & 3,09 & 2,85 \\
Cloruros (mg/l) & 3,08 & 4,12 & 3,27 & 1,41 \\
Nitratos (mg/l) & 6,30 & 0,42 & 0,51 & 0,21 \\
Sulfatos (mg/l) & 13,1 & 13,2 & 11,2 & 2,76 \\
Bicarbonato (mg/l) & 40,5 & 33,5 & 47,00 & 61,50 \\
\hline
\end{tabular}

M1 (quebrada Sanguijuela), M2 (María Aguilar), M3 (naciente San Miguel) M4 (quebrada Sardinal) 


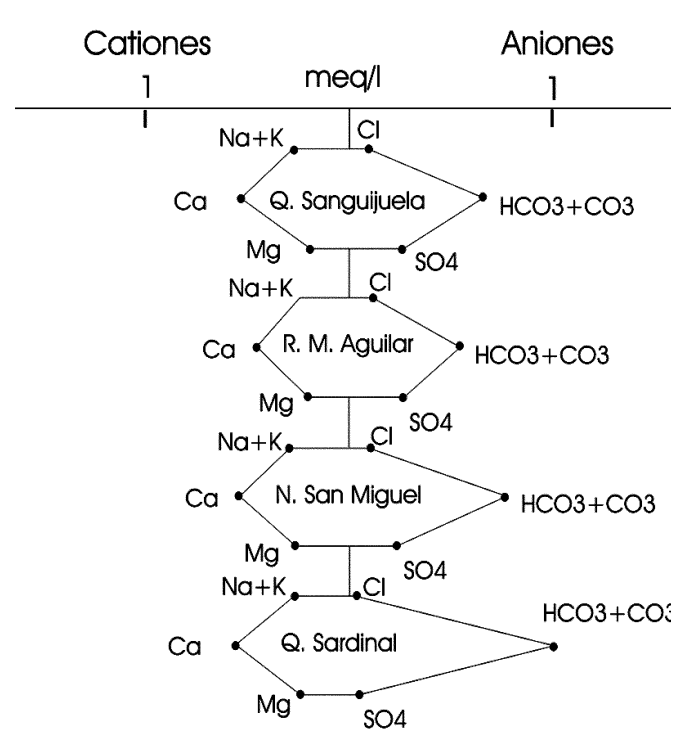

Fig. 4: Diagramas de Stiff de las muestras de agua.

sin embargo, la concentración del resto de muestras varía de 47,5 a $51,5 \mathrm{mg} / \mathrm{l}$, diferencia que se observa en los diagramas de Stiff de manera contundente (Fig. 5). Además, la concentración de calcio para la muestra de la quebrada Sanguijuela es de $13,1 \mathrm{mg} / \mathrm{l}$ mientras que el resto oscila de 8,91 a $9,87 \mathrm{mg} / \mathrm{l}$.

El día 1de noviembre de 2001 se tomaron dos muestras de agua. Una de ellas del manantial
San Miguel y otra de la perforación 40 PHC, con el fin de identificar la posible relación entre los acuíferos superior e inferior. Se analizaron los iones mayoritarios, sílice y la conductividad. El cuadro 6 muestra los valores de estos parámetros y de la alcalinidad expresada como bicarbonato. Las diferencias se notan de igual manera por medio de los diagramas de Stiff (Fig. 6). El agua del acuífero confinado tiene una concentración mayor de bicarbonato y calcio. Por otro lado, el pH tiene una ligera tendencia hacia la acidez.

\section{Ensayos de trazador}

Es probable que el agua subterránea contenida en el acuífero libre en lavas dé origen al manantial San Miguel cuando sale a la superficie. Con el fin de comprobar esta hipótesis se decidió utilizar como trazador cloruro de sodio (sal común), tomando en cuenta que éste no causa impacto en el ambiente. Además, el ion cloruro se comporta como un trazador casi ideal, ya que los cloruros son altamente solubles en agua. Si la concentración inyectada en el cuerpo de agua es grande, esta puede medirse a través de la medida de conductividad del agua. El inconveniente es que el ion cloruro siempre está presente en el agua de manera natural y algunas veces en concentraciones altas. Es deseable que el trazador se

Cuadro 5

Características químicas del agua en cinco puntos de muestreo

\begin{tabular}{|c|c|c|c|c|c|}
\hline Parámetro & M1 & M2 & M3 & M4 & M5 \\
\hline $\mathrm{pH}$ & 6,16 & 6,21 & 7,60 & 7,70 & 6,07 \\
\hline Alcalinidad como $\mathrm{HCO}_{3}(\mathrm{mg} / \mathrm{l})$ & 47,5 & 50,8 & 50,5 & 33,0 & 51,5 \\
\hline Cloruros $(\mathrm{mg} / \mathrm{l})$ & 3,40 & 1,64 & 1,33 & 3,70 & 1,59 \\
\hline Sulfatos $(\mathrm{mg} / \mathrm{l})$ & 13,0 & 1,66 & 1,39 & 15,9 & 1,27 \\
\hline Sodio $(\mathrm{mg} / \mathrm{l})$ & 5,15 & 4,48 & 3,98 & 5,72 & 4,32 \\
\hline Potasio (mg/l) & 2,82 & 2,18 & 0,93 & 2,92 & 2,00 \\
\hline Calcio $(\mathrm{mg} / \mathrm{l})$ & 9,87 & 8,91 & 9,27 & 13,1 & 9,45 \\
\hline Magnesio (mg/l) & 3,08 & 2,36 & 2,25 & 3,98 & 2,29 \\
\hline Sílice como $\mathrm{SiO}_{2}(\mathrm{mg} / \mathrm{l})$ & 54,4 & 50,7 & 45,1 & 61,9 & 52,6 \\
\hline Conductividad & 94,8 & 73,0 & 69,5 & 116,8 & 73,8 \\
\hline
\end{tabular}

M1 (naciente San Miguel), M2 (naciente No.1 de quebrada Tajo), M3 (quebrada 2), M4 (quebrada Sanguijuela), M5 (Naciente No, 2 de quebrada Tajo) 


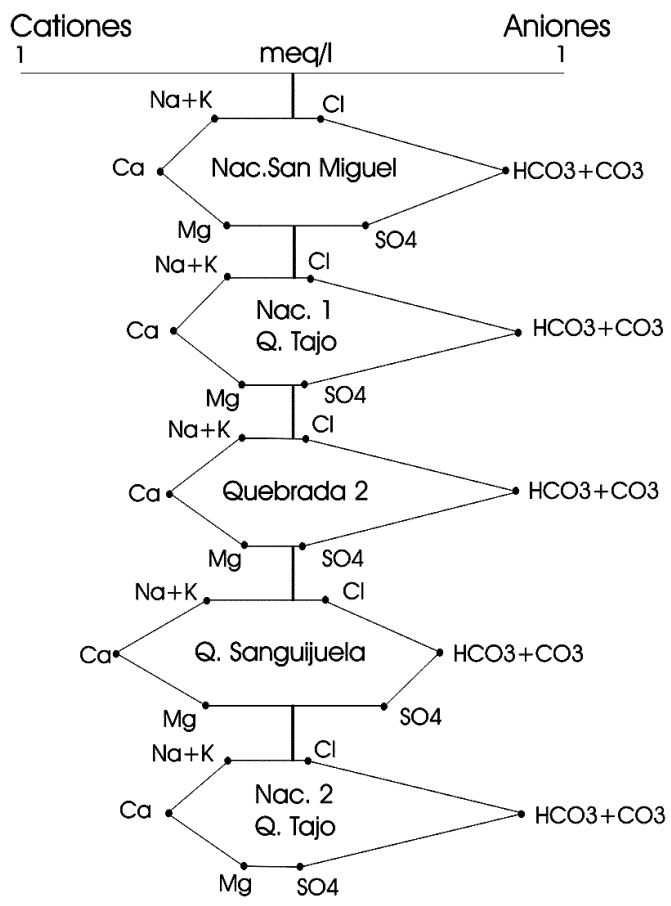

Fig. 5: Diagramas de Stiff de las muestras de agua.

Cuadro 6

Características químicas de dos muestras de agua

\begin{tabular}{ccc}
\hline Parámetro & $\begin{array}{c}\text { Naciente } \\
\text { San Miguel }\end{array}$ & $\begin{array}{c}\text { Perforación } \\
40\end{array}$ \\
\hline pH Laboratorio & 6,31 & 6,49 \\
Alcalinidad $\left(\mathrm{HCO}_{3}\right)(\mathrm{mg} / \mathrm{l})$ & 45,10 & 59,10 \\
Cloruros $(\mathrm{mg} / \mathrm{l})$ & 3,19 & 2,96 \\
Sulfatos $(\mathrm{mg} / \mathrm{l})$ & 12,00 & 11,30 \\
Sodio $(\mathrm{mg} / \mathrm{l})$ & 4,94 & 5,70 \\
Potasio $(\mathrm{mg} / \mathrm{l})$ & 2,67 & 3,22 \\
Calcio $(\mathrm{mg} / \mathrm{l})$ & 10,10 & 11,30 \\
Magnesio $(\mathrm{mg} / \mathrm{l})$ & 3,14 & 4,11 \\
Sílice $(\mathrm{SiO} 2)(\mathrm{mg} / \mathrm{l})$ & 56,70 & 69,60 \\
Conductividad $(\mathrm{mmohs} / \mathrm{cm})$ & 96,50 & 113,40 \\
\hline
\end{tabular}

utilice cuando las concentraciones naturales en el agua sean inferiores a 50 o $100 \mathrm{mg} / \mathrm{l}$ y cuando el volumen del agua a marcar sea pequeño.

El primer ensayo con el trazador se realizó el 11 de octubre del 2001 y se disolvió 45,5 kg de sal común en 185 litros de agua. Se inyectó la solución salina dentro de la perforación 37 PHC en

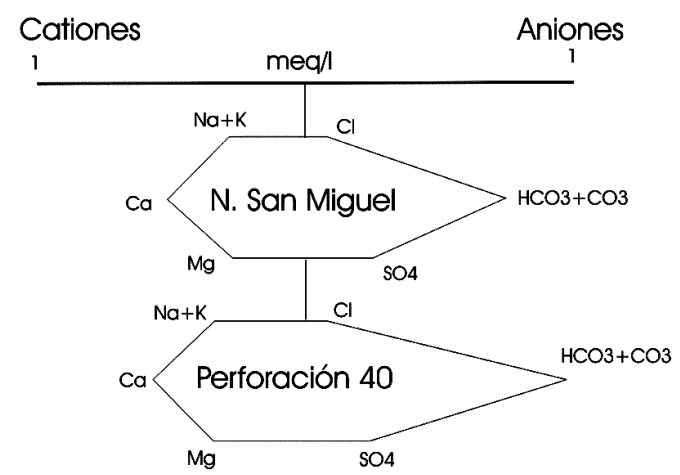

Fig. 6: Diagramas de Stiff de muestras de agua.

un período que no excedió los 20 minutos y de manera continua. Antes de iniciar la inyección se procedió a medir la conductividad del agua en el manantial San Miguel, el cual se encuentra 78 metros hacia el Noreste (Fig. 3), la cuál serviría como señal base de la conductividad. Iniciada la inyección se realizaron lecturas de conductividad en lapsos de tiempo variables hasta alcanzar un poco más de cuatro horas. Las medidas de conductividad se realizaron con un medidor de conductividad con precisión en la lectura de 0,1 $\mu \mathrm{S} / \mathrm{cm}$. Las medidas de conductividad del agua en el manantial San Miguel, en las etapas previas y durante la inyección se muestran en la figura 7. El valor máximo de conductividad se midió una hora después de la inyección y alcanzó un valor de $114,7 \mu \mathrm{S} / \mathrm{cm}$. La velocidad lineal del trazador fue de $2,6 \mathrm{~m} / \mathrm{min}$. Es necesario notar como desde la inyección hasta el valor máximo la pendiente

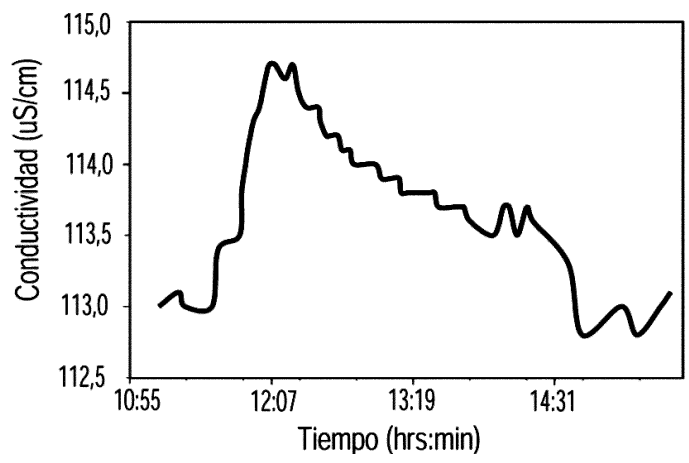

Fig. 7: Variación de la conductividad en el manantial San Miguel luego de inyectar cloruro de sodio en el acuífero superior. 
de la curva obtenida uniendo los puntos, es más pronunciada que desde el valor máximo hasta el punto final de las lecturas.

Para descartar la posibilidad de que los valores medidos de conductividad fueran producto de la variación normal de la concentración de cloruros del agua en el acuífero, se procedió a realizar un segundo ensayo el día 17 de octubre de 2001. En este se disolvieron $91 \mathrm{~kg}$ de sal común $(\mathrm{NaCl})$ en la misma cantidad de agua (185 litros) y se procedió a la inyección dentro de la perforación por medio de una manguera tal y como se hizo en el primer ensayo. De igual forma se midió la conductividad base del agua en el manantial y posteriormente se realizaron las medidas en lapsos de tiempo de 1 y 2 minutos por alrededor de 6 horas. Los resultados de las medidas realizadas se muestran en la figura 8 .

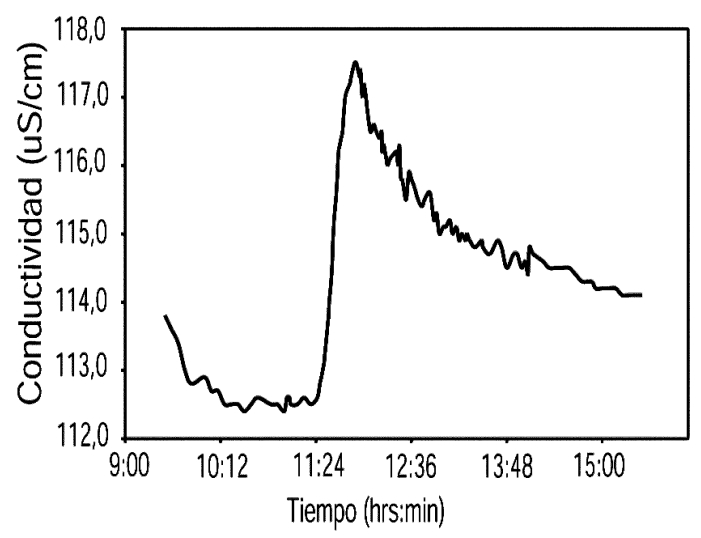

Fig. 8: Variación de la conductividad eléctrica en el manantial San Miguel durante el segundo ensayo de trazador.

En noviembre del 2001 se realizó un tercer ensayo con un volumen similar de solución, pero con la diferencia que la solución de cloruro de sodio estaba saturada. En esta prueba se adicionó otro sitio de control, la perforación 40 PHC en la cual se detectó el acuífero confinado. Se pretendía investigar si había alguna comunicación entre éste y el manantial San Miguel o con el acuífero superior en lavas. En este ensayo se ejecutaron medidas de conductividad durante 5 horas en la perforación y el manantial. Las medidas realizadas en el manantial San Miguel fueron más continuas que en las dos pruebas anteriores.
En la figura 9 se nota como la conductividad medida en el manantial ascendió después de 30 minutos de efectuada la inyección, no obstante en la perforación no se detectó mayor variación.

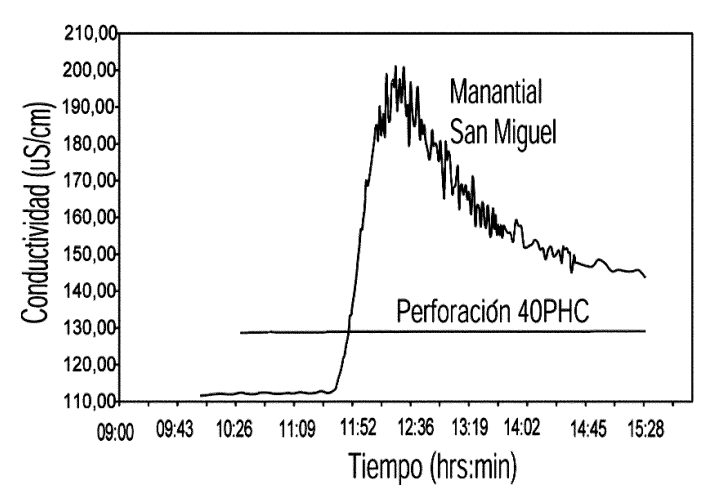

Fig. 9: Variación de la conductividad eléctrica en el agua de la perforación 40 PHC y el manantial San Miguel durante el tercer ensayo de trazador. Nótese que no hay una variación significativa en la conductividad del agua de la perforación la 40 PHC.

\section{DISCUSIÓN DE RESULTADOS}

El análisis de los resultados físico-químicos de muestras de agua recolectadas (Cuadros 4, 5 y 6), sugiere que existen varios acuíferos que dan origen a varias nacientes ya que algunas muestras presentan diferencias en las concentraciones de los elementos analizados. De hecho la quebrada Sanguijuela presentó diferencias en los bicarbonatos de hasta $20 \mathrm{mg} / \mathrm{l}$ con respecto a las otras muestras, asociado posiblemente a procesos de aireación del agua (Cuadro 4).

Por otro lado es probable que el acuífero libre en lavas descargue agua subterránea por medio de varios brotes menores. Esto se deduce al analizar los resultados del cuadro 5 correspondientes al segundo grupo de análisis ejecutado, notándose una clara relación entre el agua de los nacientes de las quebrada Tajo, la quebrada dos y el manantial San Miguel, pero lo anterior no es muy evidente con la quebrada Sanguijuela según los gráficos de Stiff (Fig. 5).

Después de inyectar la solución de cloruro de sodio en la perforación 37 PHC en tres ensayos separados, se detectó un cambio en la conductividad del agua del manantial San Miguel 
después de lapsos de tiempo que varían de 59 a 80 minutos. Este cambio en la conductividad en el manantial San Miguel demuestra que el agua subterránea que fluye en el acuífero libre en lavas encontrado en la perforación 37 PHC da origen al manantial San Miguel.

Durante el tercer ensayo con el trazador no se detectó cambio alguno en la conductividad del agua de la perforación 40 PHC durante un lapso de 5 horas desde que se inició la inyección. De estos resultados se concluye, que el acuífero libre en lavas no tiene una comunicación hidráulica con el acuífero confinado. Sumado a esto, en la perforación no se encontró el acuífero libre, más bien el agua del acuífero confiando está a presión y es surgente en éste punto.

Es evidente la importancia del manantial San Miguel como fuente de abastecimiento de agua potable para la comunidad de San Miguel de Sarapiquí y la cercanía del futuro embalse genera una interrogante sobre la posible disminución del caudal del manantial producto de la excavación del embalse.

Sin embargo, las investigaciones por medio de perforaciones realizadas en la zona, indican que el acuífero superior que origina el manantial no se extiende hasta la zona del futuro embalse. Además, el análisis de la concentración de iones mayores en el agua del acuífero libre, que da origen al manantial San Miguel y el acuífero inferior confinado captado en la perforación $40 \mathrm{PHC}$
(Cuadro 6), muestra diferencias en la conductividad y la concentración de sílice en el agua de los dos acuíferos, lo cual se observa también en los diagramas de Stiff. Por lo tanto, es poco probable que la excavación del embalse Cipreses tenga algún efecto sobre el manantial San Miguel.

\section{MODELO HIDROLÓGICO PRELIMINAR}

En la zona de estudio se presentan tres acuíferos que se recargan en las partes altas del Cerro Congo, donde los materiales presentan permeabilidades adecuadas para favorecer la infiltración y donde la precipitación es abundante. El flujo de agua dentro de los acuíferos libre y confinado se presenta por medio de fracturas y vacíos de diverso tamaño, lo que permite un tránsito expedito del agua.

El acuífero superior es de tipo libre y está constituido por lavas fracturadas de alta conductividad hidráulica. El agua subterránea que fluye por éste acuífero da origen al manantial San Miguel. El acuífero inferior es confinado y está separado del acuífero superior por materiales de menor permeabilidad. Este último podría estar presente en el sitio del futuro embalse. Un tercer acuífero de menor extensión y menor potencial está formado por arenas finas a medias y es de tipo libre (Fig. 10).

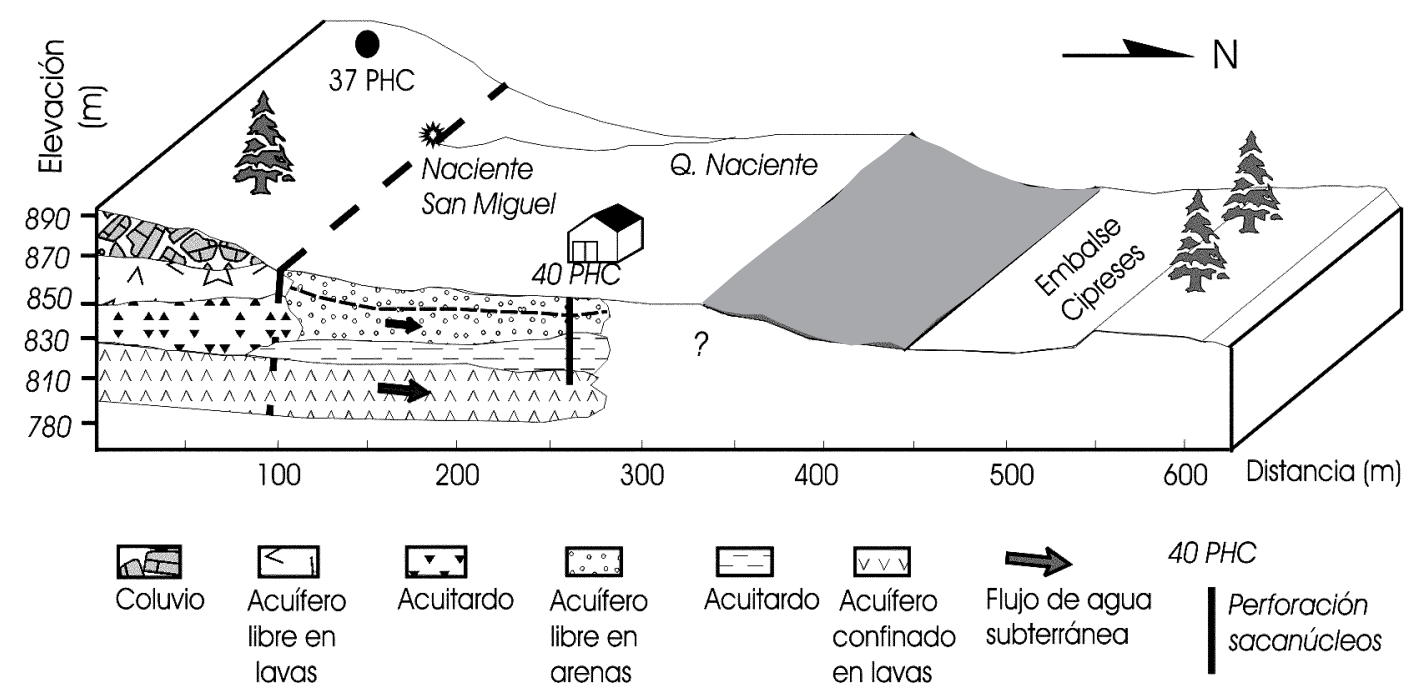

Fig. 10: Bloque diagrama mostrando la disposición de las perforaciones, el manantial San Miguel y el Embalse Cipreses. 


\section{CONCLUSIONES}

En el área de estudio se encuentran tres acuíferos constituidos por lavas y materiales epiclásticos. La descarga de agua subterránea desde el acuífero superior da lugar al manantial San Miguel, cuya zona de recarga se encuentra en las estribaciones del cerro Congo. El acuífero inferior en lavas contiene agua a presión y el nivel de agua es surgente en la perforación 40 PHC.

El análisis de los núcleos de perforación, las condiciones de presión del agua y los ensayos de trazadores indican que el acuífero que da origen al manantial San Miguel no se extiende hasta el sitio del embalse y el manantial no será afectado por la construcción del mismo.

Por la disposición espacial del acuífero superior constituido por arenas, es posible que sea cortado durante la excavación del embalse. Esta condición debe ser considerada con más detalle por medio de perforaciones en el área del embalse. Además, es necesario estudiar con más atención el efecto de la presión del agua sobre la excavación del embalse, que si bien no tiene relación directa con el acuífero superior en lavas ni con el manantial San Miguel si debe ser tomado en cuenta para efectos de presupuestos ingenieriles.

Los ensayos de trazadores han demostrado su aplicación para resolver interrogantes hidrogeológicas sin afectar en gran medida el ambiente ni la salud de la población. La aplicación de estas técnicas exige la calibración previa de los equipos (conductivímetros) y la medición continua de las conductividades en intervalos pequeños de tiempo, de lo contrario pierde su efectividad.

La ejecución de los ensayos de trazadores abre un abanico de posibilidades para el modelaje del transporte de contaminantes en el agua subterránea. Por un lado, permite estimar parámetros como el coeficiente de dispersión hidrodinámica del soluto empleado y por otro lado se pueden calibrar modelos a partir de los resultados obtenidos con la variación de la concentración del soluto.

\section{AGRADECIMIENTOS}

La implementación práctica de los ensayos de trazadores en el área de estudio estuvo a cargo del Área de Auscultación de Obras del ICE. Agradecemos a M.Sc. Rodrigo Calvo y compañeros, por el apoyo brindado.

\section{BIBLIOGRAFÍA}

ANÓNIMO, 1985: Standard methods for the examination of water and wastewater [16 ed.]. - 1268 págs. Wiley, Washington.

CUSTODIO, E. \& LLAMAS, R., 1983: Hidrología Subterránea - [2 ed.], Tomo I. 1058 págs. Omega, Barcelona.

PLATA, A., 1995: Trazadores artificiales del agua. - 33 págs. Org. Intern. Energía Atómica, Viena. 